\title{
Building Cooling Load Prediction Based on Time Series Method and Neural Networks
}

\author{
Junhua Zhuang ${ }^{1,2}$, Yimin $\mathrm{Chen}^{2}, \mathrm{Xiaoxia} \mathrm{Shi}^{2}$ and Dong Wei ${ }^{2}$ \\ ${ }^{1}$ School of Chemical Engineering \& Environment, Beijing Institute of Technology, \\ Beijing 100081, China \\ ${ }^{2}$ School of Electrical and Information Engineering, Beijing University of Civil \\ Engineering and Architecture, Beijing 100044, China \\ jpzjh@163.com
}

\begin{abstract}
Predicting the load in a building is essential for the optimal control of heating, ventilating and air-conditioning (HVAC) systems that use Ice Thermal Energy Storage (ITES) technology and also for cost and energy reduction of the non-storage systems. To solve the problems of the low accuracy of prediction by a single method, and most load predictions focusing on short-time prediction that cause reducing the practical significance, the application of the combined prediction method of time series and neural networks is presented in this paper. A case study shows that high accuracy is achieved by using the combined prediction model based on these two methods compared with the time series method in predicting the building load for longer time.
\end{abstract}

Keywords: Load Prediction, Time Series, Neural Networks, combined model

\section{Introduction}

In China, urban buildings consume more than $40 \%$ of the total electricity consumption in summer ${ }^{[1]}$. The contradiction of grid electricity supply is that the electricity supply is insufficient in daytime and superfluous in night. Thermal energy storage (TES) is an electrical load management and building equipment utilization strategy, which can reduce utility electricity demand and equipment Initial investment. Studies show that the ice thermal energy storage (ITES) has the highest efficiency ${ }^{[11]}$. Load prediction is the basis of control of the ice storage volume in off-peak price time, melting speed in peak price time and flat price time. A well-controlled ITES system should meet the following two requirements: One is that an ice storage volume must meet the requirement of air conditioning cooling in the next day. The other is that an ITES should be scheduled to release cold, and the ice in the storage device is neither exhausted ahead of schedule nor remained at the end of schedule. Therefore, an accurate prediction of the building thermal load is the basis of effective implementation of an ITES system.

Precise consumption prediction is a tough problem due to its complexity. In recent years, a large number of approaches to load prediction have been proposed and applied. Kalogirou et al. ${ }^{[15]}$ used back propagation neural networks to predict the required heating load of buildings. Ekici and Aksoy ${ }^{[2]}$ used the same model to predict building heating loads in three buildings. Minoru Kawashima et al. ${ }^{[3]}$ described an artificial neural network (ANN) model to predict the next day's total cooling load. Kreider and Wang ${ }^{[16]}$ demonstrated an automated load predictor using the ANN model. Anstett and Kreider ${ }^{[4]}$ examined the accuracy of the ANN model for energy predictions. J.R.Forrester and W.J.Wepfer ${ }^{[5]}$ firstly applied the time series method to load prediction. MacArthur et al. [6] and Spethmann ${ }^{[7]}$ developed a prediction method based on the autoregressive integrated moving average (ARIMA) model and applied it to an optimal cold storage 
controller. Support vector machines (SVM) has been increasingly used in research and industry. Li et al. ${ }^{[8]}$ used SVMs to predict the hourly cooling load of an office building.

Though there are various prediction models mentioned above, no single one has performed well enough because each model can take just several or usually only one relevant factor into consideration. For example, the time series method often considers only short-term load prediction, and it will have large deviation when considering the long-term load prediction. For above reasons, some researchers utilized combined prediction methods and achieved better results. Yao et al. ${ }^{[9]}$ proposed a method of analytic hierarchy process (AHP) that combined the four methods of time series method, linear regression (LR), grey model (GM) and artificial neural network (ANN) together. They decided the weight of each model in the combined prediction by the deviation of each model. The weight is bigger if the deviation is smaller and vice versa. They misreported the result because the data set were used repeatedly both for training and for testing. So the conclusion requires further verification.

This paper proposes a combined model of time series method and neural network to predict the ITES load by considering the periodical and tendency characteristics of the building cooling load. The periodicity and linearity of load are described by time series method, and then the residual error is described by the back-propagation (BP) neural network that can fit the nonlinear characteristic.

\section{Time Series Model}

The time series is a sequence of values $\left\{\mathrm{z}_{t}, t \in T\right\}$ obtained in accordance with an observation time. A basic feature of this sequence is a correlation among the observed values. A statistical model is fitted to the observed data by using the time series method. A common linear time series analysis includes stationary time series analysis and nonstationary time series analysis. The former includes autoregressive (AR) model, moving average (MA) model and autoregressive moving average (ARMA) models. And the latter includes autoregressive integrated moving average (ARIMA) models and seasonal models.

As the air-conditioning load shows a typical daily periodicity, even a weekly periodicity if air conditioning system is shut down or partly shut down at weekend, a seasonal model is taken into account in this paper. The seasonal model can be written as:

$$
\Phi\left(B^{s}\right) \nabla_{s}^{D} z_{t}=\Theta\left(B^{s}\right) \alpha_{t}
$$

Where, $\mathrm{z}_{t}$ is the time series of observations, and B is the backward shift operator, and $\nabla_{\mathrm{s}}=1-B^{s}$ is a difference operator of $\mathrm{s}$ horizon, and $\mathrm{D}$ is the order of the differential operator, and $\Phi\left(B^{S}\right)$ and $\Theta\left(B^{S}\right)$ are respectively the P-order polynomial and Q-order polynomial of $B^{S}$.

The model error components $\alpha_{t}, \alpha_{t-1}, \cdots$ are not generally uncorrelated. They may be written as:

$$
\phi(\mathrm{B}) \nabla^{d} \alpha_{t}=\theta(\mathrm{B}) a_{t}
$$

Where, $a_{t}$ is a white-noise process, $\phi(\mathrm{B})$ and $\theta(\mathrm{B})$ are respectively the P-order polynomial and Q-order polynomial of $\mathrm{B}$, and $\nabla=1-\mathrm{B}$ is a difference operator, and $\mathrm{D}$ is the order of it.

The seasonal model is obtained from Eq. (1) and Eq. (2) as follows:

$\phi_{p}(B) \Phi_{P}\left(B^{s}\right) \nabla^{d} \nabla_{s}^{D} z_{t}=\theta_{q}(B) \Theta_{Q}\left(B^{s}\right) a_{t}$

Eq. (3) is often called (p,d.q) $\times(P, D, Q)$.

\section{Artificial Neural Networks (Ann) Model}

Neural network has the ability to approximate any function, including non-linear functions. A typical neural networks used in the present study is shown in Fig, 1. This is 
called feed forward type of network where computations proceed along the forward direction only. There are three layers of neurons, namely input, hidden and output layer. The output obtained from the output neurons constitutes the network output.

A neural network has $\mathrm{n}$ input nodes, $\mathrm{m}$ output nodes and $\mathrm{p}$ hidden nodes. The each hidden node works as the following equation:

$$
\mathrm{x}_{j}=f\left(\sum_{i=1}^{n} w_{i j} x_{i}+b_{j}\right), j=1,2, \cdots, p
$$

The each output node works as the following equation:

$$
\mathrm{y}_{k}=\sum_{j=1}^{p} w_{j k} x_{j}+b_{k}, k=1,2, \cdots, m
$$

Where, $x_{i}$ is the input values; $x_{j}$ is the output from the neurons of hidden layer; $y_{k}$ is the neural network outputs; $w_{i j}, w_{j k}$ are the connection weights; $b_{j}, b_{k}$ are the bias values; $f$ is the transfer function, typically sigmoidal function given by

$$
f(x)=\frac{1}{1+e^{-\mu x}}
$$

The connection weights and bias values are initially chosen as random numbers and then fixed by the results of a training process. Many alternative training processes are available, from which the study adopted a popular scheme named back-propagation (BP). The goal of any training algorithm is to minimize the global error $E$ (mean sum squared), which defined below:

$$
E=\frac{1}{2} \sum\left(O_{n}-t_{n}\right)^{2}
$$

Where, $O_{n}$ and $t_{n}$ are network and target output for any $n$th output node. The summation has to be carried out over all output nodes for every training pattern; a pair of input and output values constitutes a training pattern.

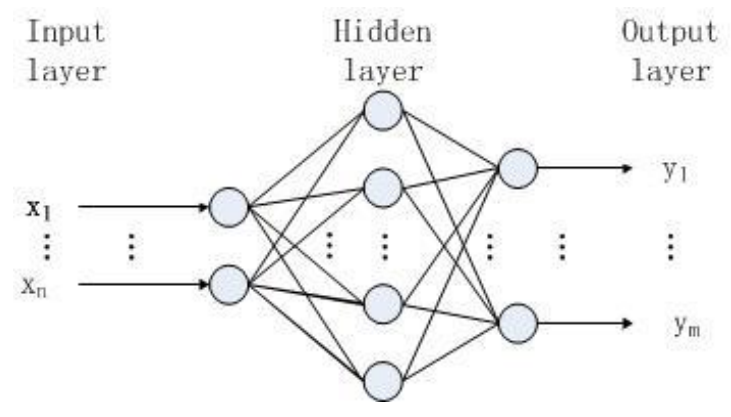

Figure 1. A Typical BP Neural Network

\section{The Combined Model}

Time series of building cooling load usually have complex linear and nonlinear characteristics. This complex features cannot be well characterized in a single seasonal model. Furthermore, various results can be caused by using neural network model to linear problems. Markham and Rakes ${ }^{[10]}$ found that the performance of neural networks for linear regression problems depends on the sample size and noise level. Although neural network has the ability to approximate any function, it is complicated for fitting functions include linear component, non-linear component, cyclical component and trend component. Currently, there are few methods to determine the number of hidden layers and hidden layer neural network nodes except of trial and error method. Thus, it is necessary to reduce the complexity of functions that is fitted by neural networks.

Assuming the time series $z_{t}$ can be seen as a combination of a linear part $L_{t}$ and nonlinear part residuals $N_{t}$ :

$$
z_{t}=L_{t}+N_{t}
$$


Firstly, the linear component (includes Cyclical component and Trend component) of the time series is modeled by a seasonal model. Let prediction result is $\hat{\mathrm{L}}_{t}$, then the residual of the sequence $\mathrm{z}_{t}$ and $\hat{\mathrm{L}}_{t}$ ise $\mathrm{e}_{t}$. It can be written as:

$$
\mathrm{e}_{t}=z_{t}-\hat{L}_{t}
$$

The sequence $e_{t}$. Contains the nonlinear information of the original sequencez $t_{t}$, and the non-linear relationship can be fitted by a neural network. If the neural network has $\mathrm{n}$ inputs, the residual sequence $\mathrm{e}_{t}$ can be written as:

$$
\mathrm{e}_{t}=f\left(e_{t-1}, e_{t-2}, \cdots, e_{t-n}\right)+\varepsilon_{t}
$$

Where, $f$ is the nonlinear function fitted by the neural network, $\varepsilon_{t}$ is the random error and also the entire combined model error. If the residuals $e_{t}$ predicted by the neural network is denoted as $\widehat{\mathrm{N}}_{t}$, the combined model predicted results can be written as follows:

$$
\hat{z}_{t}=\widehat{L}_{t}+\widehat{N}_{t}
$$

The combined model uses the seasonal time series method to fit the trend, cycle, linear components, and uses neural network to fit nonlinear component. It both improves the prediction accuracy, and reduces the complexity of the neural network.

\section{The Load Prediction by Using the Combined Model}

For further illustrate the performance of the proposed combined model, an example of hourly cooling load prediction for an office building is presented. Because the building is just put into operation, the real load data is obtained by DeST, which is software for the building load calculations, instead of the multiplication of water flow and the temperature difference between inlet and outlet water. An effective control strategy based on load prediction should be applied in time when a building is put into use. Thus, it is meaningful for the data obtained by DeST.

The load prediction is modeled by using the data from July 1 to July 31 , and then the load of the first week of August is predicted, and is compared with the real data in order to evaluate the model effectiveness.

\subsection{Removing Periodicity of Load}

A week of samples is drawn from the data to be drawn a timing diagram Figure 2. The real load presents an obvious periodicity of 24 hours (daily periodicity). Figure 3 is the load 24 order difference, which removes the periodicity. Figure 4 shows the load difference of the whole July.

\subsection{Model Identification by Using Time Series Method}

5.2.1. Stationary Test: From Figure4, periodicity and trend cannot be seen obviously. Figure 5 is the stationary test of load data that is removed periodicity. From it, the autocorrelation coefficient of the sequence is not fast decay toward zero when delay steps increase. It means the sequence is a non- stationary sequence.

But the 1, 2 or 3 order-difference autocorrelations of the sequence indicates that the sequences have large autocorrelation coefficients when delay steps are large, which means difference could not eliminate the non- stationary. Although in theory, a sufficient number of differential operations can extract the non-stationary information from the sequence, a differential operation is a process of extracting information, which will result in the loss of information. Finally, the sequence without a differential operation is chosen after several tests. 




Figure 2. Timing Diagram of a Week of Samples

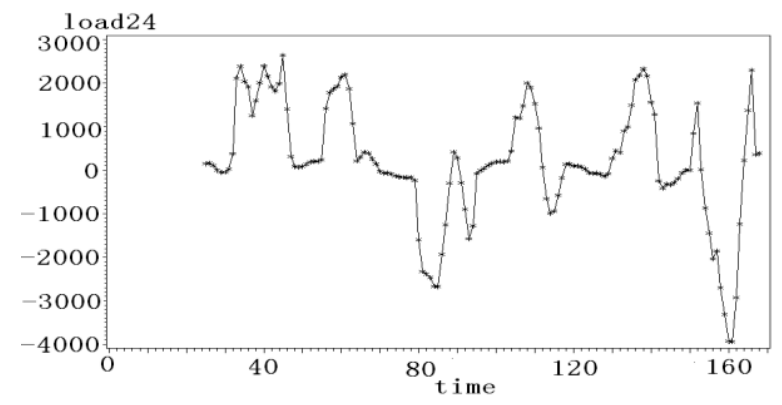

Figure 3. The Load Difference of a Week of Samples

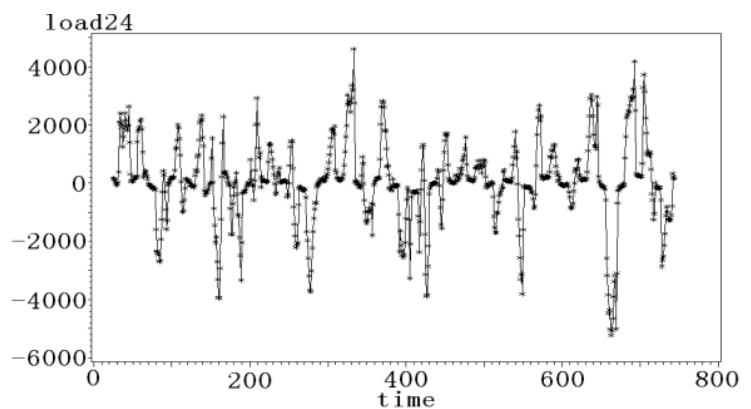

Figure 4. The Load Difference of the Whole July

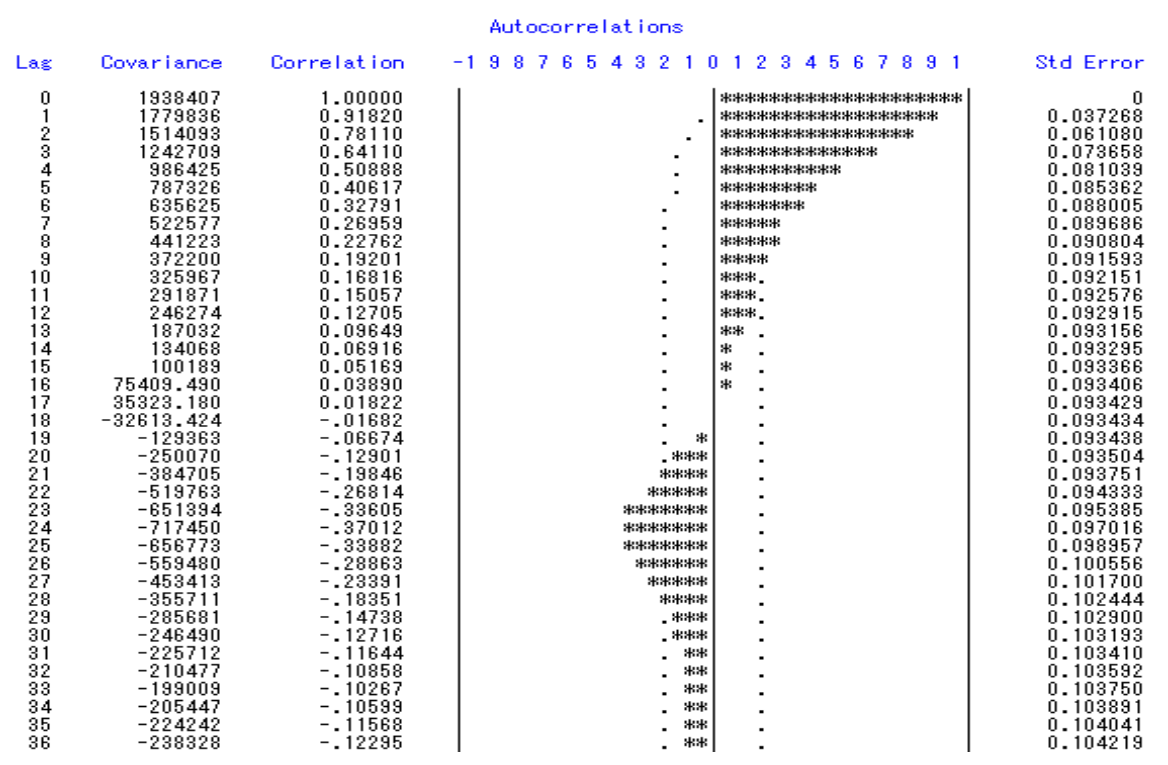

Figure 5. The Autocorrelations Diagram of the Sequence 
5.2.2. Model Order, Parameter Estimation and Model Testing: Statistics Analysis System (SAS), statistical software, can be used to determine the order of the model and to estimate the model parameters by using the ESTIMATE command of ARIMA process, which uses maximum likelihood estimation (MLE) method or least-square estimation (LSE) method. A sequence can often be fitted by more than one model when identifying models. Although the properties of truncation and tailing of autocorrelation and mutualcorrelation can be used to identify the model, the hybrid model that includes autoregressive (AR) model and moving average (MA) model still cannot obtains satisfactory outcome. It is common, because the model identification is often tentative. Using the AIC and SBC criteria, the models that have smaller value of these criteria should be chosen. Note the method of model identification must be MLE [12-14].

After determining the model, model test is also necessary. Model test includes significance test of parameters and white noise test of residuals. Parametric test is to examine whether each of the unknown parameters were significantly non-zero or not, the purpose is to make the model simpler. The test uses $t$ distribution test, and removes the independent variables related to non-significant parameters, and then construct a simpler model. Residuals should be a white noise, which indicate that they no longer contain any relevant information. Hence residuals should be verified of the white noise by using $\chi^{2}$ distribution.

Some model structures, AIC and SBC values, identified parameters and the white noise test results of the seasonal differential load data of July are listed below.

(1) $\mathrm{p}=(1), \mathrm{q}=(1,2,3), \mathrm{AIC}=11012.57, \mathrm{SBC}=11035.46$, parameters are all significant by testing. The white noise test of residuals is significantly effective when the delay interval of correlation is 6,12 or 18 , and is not significantly effective when the delay interval has bigger value.

(2) $\mathrm{p}=0, \mathrm{q}=(1,2,3), \mathrm{AIC}=11192.73, \mathrm{SBC}=11211.04$, parameters are all significant by testing. The white noise test of residuals is not significantly effective when the delay interval takes any value.

(3) First difference, $\mathrm{p}=0, \mathrm{q}=(1,24), \mathrm{AIC}=10808.27, \mathrm{SBC}=10822.01$, parameters are all significant by testing. The white noise test of residuals is not significantly effective when the delay interval takes any value.

(4) first difference, $\mathrm{p}=(1,2,4), \mathrm{q}=0, \mathrm{AIC}=11039.76, \mathrm{SBC}=11058.07$, parameters are all significant by testing. The white noise test of residuals is significantly effective when the delay interval of correlation is 6 , and is not significantly effective when the delay interval has a bigger value.

(5) Double difference, $\mathrm{p}=4, \mathrm{q}=1$. $\mathrm{AIC}=11232.46, \mathrm{SBC}=11259.92$, parameters are all significantly by testing. The white noise test of residuals is not significantly effective when the delay interval takes any value.

(6) Double difference, $\mathrm{p}=3, \mathrm{q}=0$. $\mathrm{AIC}=11229.91, \mathrm{SBC}=11248.22$, parameters are all significantly by testing. The white noise test of residuals is not significantly effective when the delay interval takes any value.

None of the mean MU in above models is significantly effective, and significant models cannot be obtained after many tests.

Considering the AIC and SBC values, parameters and white noise test results, the No.1 model is chosen, which is $\mathrm{p}=(1), \mathrm{q}=(1,2,3)$. The formula describes the model as:

$$
\left(1-\phi_{1} \mathrm{~B}\right)\left(1-B^{24}\right)\left(z_{t}-\mu\right)=\left(1-\theta_{1} B-\theta_{2} B^{2}-\theta_{3} B^{3}\right) a_{t}
$$

The MLE of the model parameters are estimated as: $\phi_{1}=0.79375 ; \theta_{1}=-0.50393$; $\theta_{2}=-0.18871 ; \theta_{3}=-0.16131 ; \mu=52.44734$.

5.2.3 Model Prediction: The application of load prediction in ITES is considered in this paper. The hourly load of next day must be predicted before ice charging in the night, and the short-term (e.g. hourly) load prediction is useless. The next 24 hours load predictions 
of 24:00 Aug. 1 and comparison with the real value are showed in Figure 6. The prediction uses the Eq. 12.

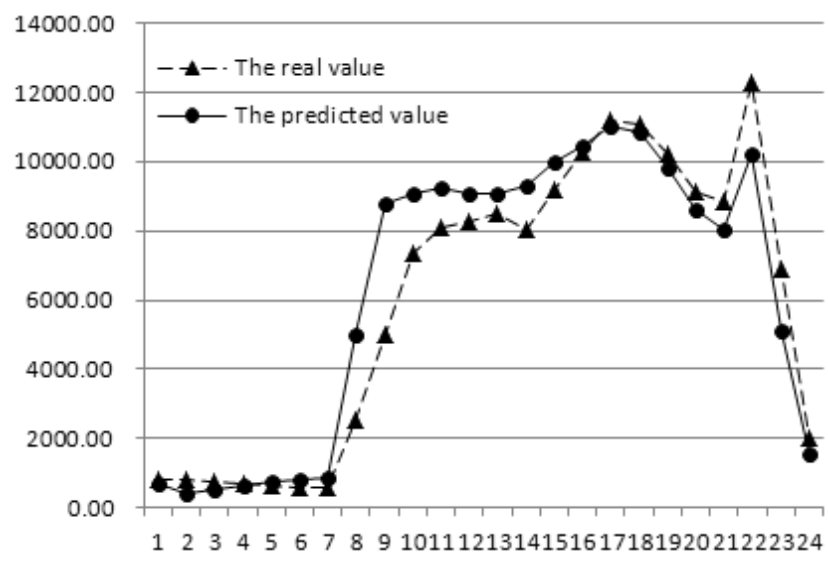

Figure 6. The Next 24 Hours Load Predictions

Although FORCAST command of ARIMA in SAS can make multi-steps prediction, the command only makes multi-steps prediction at the end of the data, and makes singlestep prediction with most data in the process. This is enough for the practical applications, but does not meet the needs of the research, because researchers must learn the accuracy of prediction and the credibility of the model through analyzing a large number of multisteps prediction accuracy.

The problem has resolved by writing formulas of the model in Excel. The initial value of $a_{t}$ in the model must be well dealt with. Using forward recursion and backward recursion method introduced in ${ }^{[12]}$, the initial value can be well obtained. The result of single-step prediction using Excel is similar with the result using SAS, and the difference is within $1 \%$.

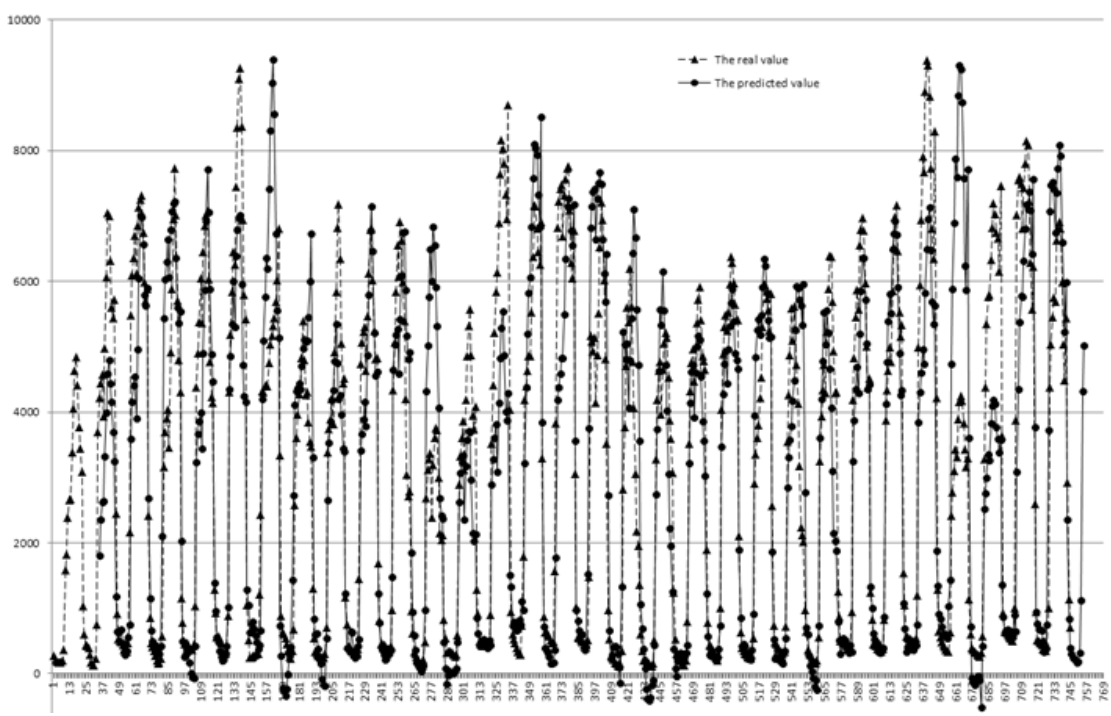

Figure 7. The Result of 10 Steps Prediction

Using 10 steps (10 hours) prediction as an example. Figure7 shows the result of daily prediction in July. It is note that it is wrong to analyze the model validity by using the data of July, since the model is based on the same data. The correct approach uses 
different data to test the validity. The wrong approach mentioned above will be stated for further analysis by using neural networks.

\subsection{TES Load Prediction Based On the Combined Model}

The nonlinear component of the sequence $\mathrm{e}_{t}$ is recognized as the result $\widehat{N}_{t}$ by a BP neural network. It is difficult to determine the number of network input nodes and hidden nodes for constructing the model. There is no theoretical guidance, but only experience, trial and error. The neural networks are trained by using the data as Figure7 shows. By constantly trying to change the number of nodes, the convergence speed and the ability to fit the model is improved. This process will not be stopped until the network output error meets the requirement. After repeated trials, the network is determined the 3 input 1 output network (predicting $\mathrm{e}_{t}$ by usinge $\mathrm{e}_{t-1}, \mathrm{e}_{t-2}, \mathrm{e}_{t-3}$ ), and the number of hidden nodes is 4 . The network is a $3 \times 4 \times 1$ network. Network test (using) use the first week load data of August. Figure 8 shows the normalized results of the residuals predicting error.

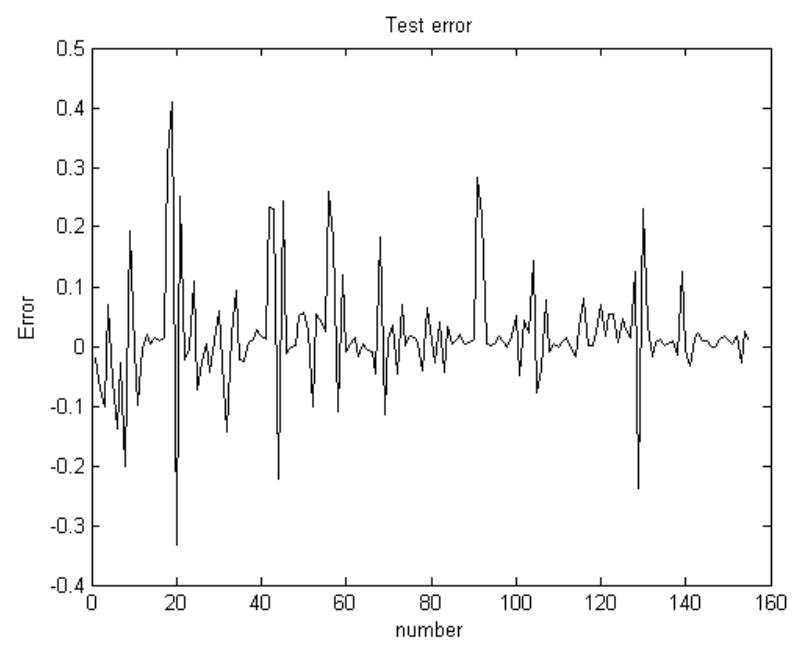

Figure 8. The Normalized Results of the Residuals Predicting Error

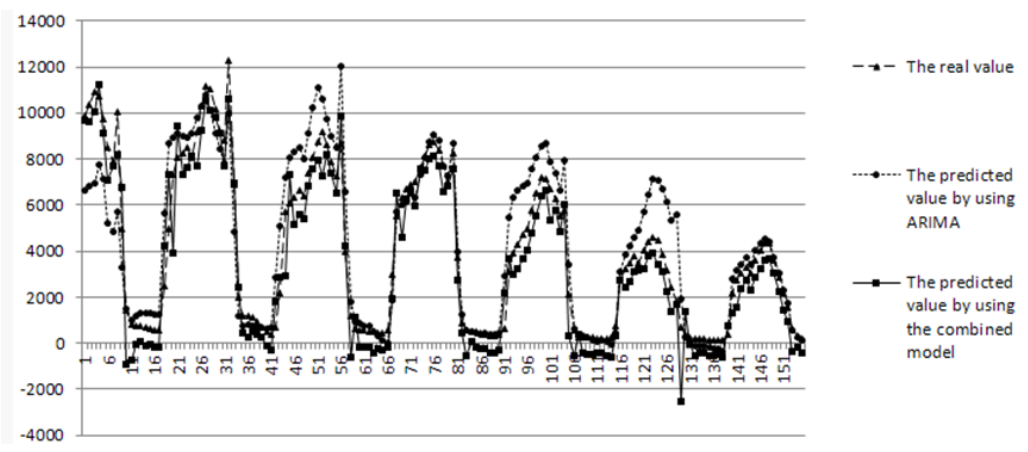

Figure 9. Comparison of the Real Value and Predicted Values by Two Methods

The residuals predicted by neural network may firstly be anti-normalized, and then add the predicted value by time series method to obtain a final prediction. Figure 9 shows the comparison of the real value, predicted value by ARIMA and predicted value by ARIMA+NN of the first week load data of August. AS a result, the predicting accuracy by using the combined model is higher than by using single time series method. The difference between the predicted value and the real value is small, so the prediction method is of practical value. 


\section{Conclusion}

In this paper, the TES load data is modeled by using the seasonal model firstly, which focuses on the analysis of the periodicity of the sequence. Then the residuals of ARIMA are modeled by a neural network. The combined model is superior to simple time series model, and effectively solves the problem of fitting nonlinearity caused by using time series method. In practice, the short-term load prediction, e.g. hourly prediction, does not make sense for the ice charging process in the ITES system. This paper uses 10-hour prediction as an example, and discusses the methods and results of longer-term prediction. The results show the combined method is effective.

In general, in the load prediction, meteorological parameters such as temperature, humidity and illumination are often taken by using neural networks as inputs and the load prediction is conducted as output. Meteorological parameters is not concerned in this paper. The research will explore the time series model with input (ARIMAX), and combine it with neural networks, and then compare the three methods: ARIMAX, neural networks and ARIMAX-neural networks.

\section{References}

[1] Z. G. Hu, "Application of Demand Side Management to Peak load leveling in Beijing, Electric Power", vol. 31, no. 9, (1998), pp. 37, (in Chinese).

[2] B. B. Ekici, U. T. Aksoy, "Prediction of building energy consumption by using artificial neural networks", Advances in Engineering Software, vol. 40, no. 5, (2009), pp. 356-62.

[3] M. Kawashima, C. E. Dorgan, et al., "Optimizing system control with load prediction by neural networks for an ice-storage system", ASHRAE Trans. Part 1, vol. 102, (1996), pp. 1169-1178.

[4] M. Anstett and J. F. Kreider, "Application of neural networking model to predict energy use", ASHRAE Trans. Part, vol. 1, no. 99, (1993), pp. 505-517.

[5] J. R. Forrester and W. J. Wepfer, "Formulation of a load prediction algorithm for a large commercial building", ASHRAE Trans, vol. 90, (1984), pp. 536 - 551.

[6] J. W. MacArthur, A. Mathur and J. Zhao, "On-line recursive estimation for load profile prediction", ASHRAE Trans, Part 1, vol. 95, (1989), pp. 621-628.

[7] D. H. Spethmann, "Optimal control for cool storage", ASHRAE Trans. Part 1, vol. 95, (1989), pp. 710 721.

[8] Q. Li, Q. L. Meng, J. J. Cai, Y. Hiroshi and M. Akashi, “Applying support vector machine to predict hourly cooling load in the building", Applied Energy, vol. 86, no. 10, (2009), pp. 2249-56.

[9] Y. Yao, Z. Lian, S. Liub and Z. Hou, "Hourly cooling load prediction by a combined forecasting model based on Analytic Hierarchy Process", International journal of thermal sciences, vol. 43, (2004), pp. $1107-1118$.

[10] I. S. Markha and T. R. Rakes, "The effect of sample size and artificial neural networks and regression", Computers \& Operation Research, vol. 25, no. 4, (1992), pp. 251-63.

[11] H. Yu, "The Technology and Design of Ice Thermal Energy Storage", Chemical Industry Press, (2007), (in Chinese)

[12] G. E. P. Box, G. M. Jenkins, G. C. Reinsel, translated by C. Z. Wang, M. F. You and Y. Hao, "Time Series Analysis", Forecasting and Control, China Machine Press, (2011), (in Chinese).

[13] Y. Wang, "Applied Time Series Analysis, China Renmin University Press", (2012), (in Chinese).

[14] F. Wang, S. K. Chen and G. S. Feng, "Statistical Analysis and Application Based on SAS", Publishing House of Electronics Industry, (2011), (in Chinese).

[15] S. A. Kalogirou, C. C. Neocleous and C. N. Schizas, "Building heating load estimation using artificial neural network", Proceedings of the 17th international conference on parallel architectures and compilation techniques, (1997).

[16] J. F. Kreider and X. A. Wang, "Improved artificial networks for commercial building energy use prediction", Proceedings of the ASME Annual Solar Engineering Meeting, Maui, HI, (1992), pp. 361366. 


\section{Authors}

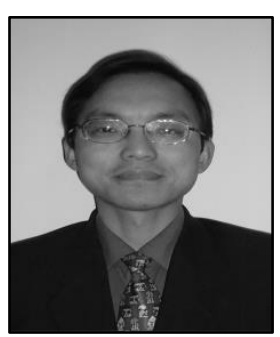

Junhua Zhuang

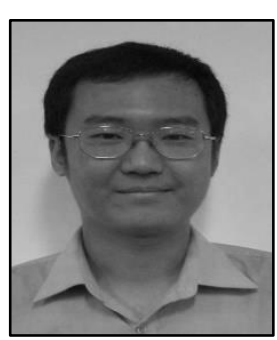

\section{Yimin Chen}

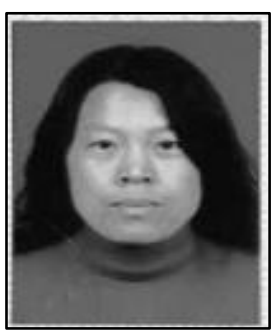

Xiaoxia Shi



Dong Wei 